\title{
Vigilância e busca ativa de casos suspeitos de dengue hemorrágico em Ribeirão Preto, São Paulo'
}

\author{
Ricardo José Soares Pontes ${ }^{2}$ e Antônio Ruffino-Netto ${ }^{3}$
}

RESUMO Um estudo clínico-epidemiológico, do tipo relato de casos, avaliou o sistema de vigilância e busca ativa de casos suspeitos de dengue hemorrágico/sindrome de choque do dengue $(\mathrm{DH} / \mathrm{SCD})$ durante uma epidemia de dengue no Município de Ribeirão Preto, Estado de São Paulo, Brasil, que ocorreu entre novembro de 1990 e março de 1991. Tratou-se da primeira epidemia de dengue registrada nessa região. Embora o dengue seja um problema de saúde pública crescente no Brasil e o DH/SCD pareça tratar-se de um problema em evolução nas Américas, os serviços de saúde pública demonstram uma atitude passiva diante da ocorrência da doença, em parte como fruto da falta de experiência. A divulgação da ocorrência desta enfermidade, tanto na forma de casos esporádicos como na forma epidêmica, é essencial para a redução da letalidade.

Os dados utilizados na presente análise foram coletados pelo sistema oficial de vigilância epidemiológica do Município de Ribeirão Preto durante o período da epidemia. Entre 2521 casos confirmados de dengue foram identificados 36 casos suspeitos de DH/SCD 34 casos de doença febril aguda com manifestações hemorrágicas e 2 óbitos associados ao dengue. Desse conjunto, 12 casos de dengue com manifestações hemorrágicas foram confirmados laboratorialmente. A análise dos dados clínicos, epidemiológicos, laboratoriais e de autópsia dos óbitos associados ao dengue sugeriu tratarem-se de casos de DH/SCD primário. O estudo aqui descrito evidenciou a dificuldade do sistema assistencial de saúde em estabelecer a suspeita clínica de DH/SCD. Isto acarretou demora e inadequação na conduta clínica. Além disso, ficou confirmada a necessidade de um sistema de vigilância epidemiológica ativa permanente, que possibilite a identificação precoce dos casos suspeitos de DH/SCD e viabilize uma ação de controle rápida e adequada.

1 Trabalho extraído de tese de doutorado intitulada "Estudo da epidemia de dengue no município de Ribeirão Preto - SP, 1990-1991", apresentada em dezembro de 1992 ao Departamento de Medicina Social, Faculdade de Medicina de Ribeirão Preto da Universidade de São Paulo.

2 Universidade Federal do Ceará, Departamento de Saúde Comunitária, Centro de Ciências da Saúde, Rua Professor Costa Mendes, 1608, $5^{\circ}$ andar/ Rodolfo Teófilo, CEP 60431-970, Fortaleza, Ceará, Brasil. Correspondência e pedidos de separatas devem ser enviados a este endereço.

3 Faculdade de Medicina de Ribeirão Preto da Universidade de São Paulo, Ribeirão Preto, SP, Brasil.
O dengue é uma arbovirose da maior importância em saúde pública. Em sua etiologia estão envolvidos quatro sorotipos virais da família Flaviviridae, denominados DEN-1, DEN2, DEN-3 e DEN-4 (1,2). Clinicamente, os casos da doença apresentam-se dentro de um amplo espectro, assim distribuído: a) infecção assintomática ou febre indiferenciada; b) quadro típico da síndrome de febre do dengue ou dengue clássico; c) febre hemorrágica do dengue ou dengue hemorrágico/ síndrome de choque do dengue (DH/SCD). O dengue clássico pode apresentar, em alguns casos, fenômenos hemorrágicos menores (como petéquia, epistaxe, gengivorragia, entre outros), tornando necessário o diagnóstico diferencial com o $\mathrm{DH} /$ 
SCD. A Organização Mundial da Saúde (OMS) estabelece critérios clínicos e laboratoriais para a classificação dos casos de DH/SCD, de modo a diferenciá-los dos casos de dengue com manifestações hemorrágicas (2).

Observações epidemiológicas e laboratoriais sugerem que a infecção secundária ou seqüencial heterotípica representa o principal fator de risco para a ocorrência do DH/SCD, particularmente na forma epidêmica ou endemo-epidêmica (3, 4). Entretanto, podem acontecer casos de $\mathrm{DH} / \mathrm{SCD}$ em infecções primárias. Isto se observa em crianças menores de um ano portadoras de anticorpos maternos contra o dengue (passados por via transplacentária) e envolve um mecanismo fisiopatológico semelhante ao da infecção seqüencial $(5,6)$. Além disso, casos de $\mathrm{DH} / \mathrm{SCD}$ têm sido relatados em áreas sem circulação anterior do dengue, ou em locais onde a enfermidade esteve ausente por várias décadas (7-9), embora este fenômeno seja relativamente raro e fuja à explicação etiopatogênica da infecção seqüencial. É possível que cepas mais virulentas ou fatores de risco individuais (por exemplo, idade, sexo, raça, doenças crônicas) participem da gênese do DH/SCD em pessoas primo-infectadas $(10,11)$.

Entre novembro de 1990 e março de 1991 ocorreu uma epidemia de dengue no Município de Ribeirão Preto que se irradiou para outros municípios do Estado de São Paulo. Tratou-se da primeira epidemia de dengue nesta região geográfica do Estado. A circulação do sorotipo DEN-1 foi identificada (12). Durante a epidemia chamou a atenção a ocorrência de casos clinicamente suspeitos de dengue com a presença de fenômenos hemorrágicos, geralmente leves. O sistema de vigilância epidemiológica, alertado para a possibilidade de casos de DH/SCD, organizou, a partir da primeira semana da epidemia, um processo de vigilância e busca ativa de casos suspeitos de DH/SCD que abrangia todos os serviços de saúde do Município de Ribeirão Preto.

O presente trabalho pretende relatar e discutir o processo de identificação, pelo sistema de vigilância e busca ativa, dos casos suspeitos de dengue hemorrágico e, especialmente, dos óbitos associados ao dengue durante o processo epidêmico em Ribeirão Preto.

\section{MATERIAIS E MÉTODOS}

O presente trabalho apresenta um estudo clínico-epidemiológico descritivo, do tipo relato de casos. Os dados utilizados foram coletados pelo sistema oficial de vigilância epidemiológica do Município de Ribeirão Preto entre novembro de 1990 e março de 1991, durante uma epidemia de dengue.

O Município de Ribeirão Preto situase na região noroeste do Estado de São Paulo, Brasil. Ribeirão Preto é um pólo econômico regional que centraliza um intenso intercâmbio de atividades com municípios localizados em sua área geográfica de influência, além de ser considerado um dos principais centros de pesquisa e ensino biomédico do país. A rede assistencial pública de saúde é de boa qualidade e oferece cobertura populacional considerada alta para os padrões brasileiros. Em 1990, a população do Município de Ribeirão Preto era de 452746 habitantes, conforme a Secretaria do Planejamento de Ribeirão Preto. Durante a epidemia de dengue em 1990-1991, 8963 casos foram notificados às autoridades sanitárias, dos quais 2521 foram confirmados laboratorialmente (12). Deste conjunto, o presente estudo focalizou os 36 casos identificados como suspeitos de DH/SCD através do sistema de vigilância e busca ativa organizado durante a epidemia. Esses casos foram subdivididos em dois grupos, de acordo com fontes e tipo de dados coletados: a) casos ambulatoriais suspeitos de $\mathrm{DH} / \mathrm{SCD}$; b) casos hospitalizados suspeitos de DH/SCD.

\section{Casos ambulatoriais}

Durante a epidemia, os casos de febre com manifestações hemorrágicas atendidos nas unidades ambulatoriais do setor público do Município foram encaminhados para um ambulatório de referência organizado pelo sistema de vigilância epidemiológica, onde os pacientes eram submetidos a uma avaliação clínica preliminar e exames laboratoriais, entre eles exame sorológico para dengue, prova do laço, contagem de plaquetas e hematócrito. Por razões de ordem administrativa, esse serviço funcionava somente de segunda a sexta-feira, das 7 às 17 horas. Por esse sistema de vigilância foram identificados 33 casos ambulatoriais suspeitos de $\mathrm{DH} / \mathrm{SCD}$.

As informações referentes aos casos ambulatoriais suspeitos de dengue que apresentavam manifestações hemorrágicas foram coletadas em fichas de investigação epidemiológica do dengue, preenchidas pelo setor de vigilância epidemiológica da Secretaria Municipal de Saúde de Ribeirão Preto. Essas fichas registravam o nome do paciente, local de moradia, data da notificação e primeiros sintomas, idade, sexo, tipo de sangramento ocorrido e os resultados dos exames laboratoriais. Os exames laboratoriais de todos os casos estudados foram realizados no Instituto Adolfo Lutz, da Secretaria de Saúde do Estado de São Paulo. Na sorologia para dengue foi utilizada a técnica MAC-ELISA para captura de IgM.

\section{Pacientes hospitalizados}

Ao mesmo tempo em que acionava as unidades ambulatoriais, o sistema de vigilância epidemiológica ficou alerta para a ocorrência de casos ou óbitos compatíveis com DH/SCD na rede hospitalar do Município. Um processo de busca ativa para casos hospitalares suspeitos foi organizado, através de contatos periódicos por parte da equipe de vigilância epidemiológica com equipes médicas, equipes de enfermagem e serviço de microscopia. A partir deste processo foram identificados três casos hospitalizados suspeitos de DH/SCD, dois dos quais com evolução para óbito.

Os dados sobre a história clínica dos casos hospitalizados suspeitos de DH/SCD foram obtidos nos prontuários médicos do Hospital de Clínicas de Ribeirão Preto e da Santa Casa de Ribeirão Preto. A investigação epide- 
miológica destes casos foi realizada pelos setores de vigilância epidemiológica do Escritório Regional de Saúde de Ribeirão Preto/Secretaria de Saúde do Estado de São Paulo e da Secretaria Municipal de Saúde de Ribeirão Preto, além do Centro de Pesquisa e Vigilância Epidemiológica do Departamento de Medicina Social (Faculdade de Medicina de Ribeirão Preto/Universidade de São Paulo). Os exames necroscópicos dos óbitos associados ao dengue foram realizados pelo Serviço de Verificação de Óbitos do Hospital de Clínicas de Ribeirão Preto. Os exames imunohistoquímicos de fragmentos de tecidos e a sorologia para dengue foram feitos pelo Instituto Adolfo Lutz da Secretaria de Saúde do Estado de São Paulo, como parte da investigação epidemiológica dos casos. Na sorologia para dengue foi utilizada a técnica MAC-ELISA para captura de IgM.

\section{Caracterização de DH/SCD}

Os critérios utilizados para a caracterização dos casos de DH/SCD foram aqueles recomendados pela OMS (2): hemoconcentração (aumento de $20 \%$ no hematócrito); evidências de aumento da permeabilidade vascular (efusão de plasma); trombocitopenia (menor ou igual a 100 000/ $\mathrm{mm}^{3}$ ); evidências laboratoriais de infecção pelo vírus do dengue; e quadro clínico compatível, onde se destaca a existência de uma doença febril aguda seguida por choque hipovolêmico ou por fenômenos hemorrágicos. Na ausência de dois hematócritos seriados, as faixas de normalidade a seguir discriminadas foram utilizadas: homens $=46 \%(36 \mathrm{a}$ $52 \%)$; mulheres $=41 \%$ (35 a $47 \%)$; crianças $=35$ a $49 \%$.

\section{RESULTADOS}

No período epidêmico foram confirmados, por critérios clínicolaboratoriais, um total de 2521 casos de dengue, assim distribuídos: 98 casos em novembro; 222 em dezembro; 171 em janeiro; 28 em fevereiro; e 2 em março. Nesse mesmo período, foram notificados 33 casos ambulatoriais clinicamente compatíveis com dengue e que apresentavam manifestações hemorrágicas, sendo considerados por este motivo suspeitos de $\mathrm{DH} / \mathrm{SCD}$ (20 casos em dezembro; $7 \mathrm{em}$ janeiro; 4 em fevereiro; e 2 em março). Além disso, 1 caso e 2 óbitos de pacientes hospitalizados foram identificados como suspeitos de DH/SCD (o primeiro em novembro, outro em dezembro e o terceiro em janeiro).

O quadro 1 apresenta as principais informações disponíveis a respeito dos casos ambulatoriais suspeitos de $\mathrm{DH} / \mathrm{SCD}$. A figura 1 mostra a distribuição no tempo dos casos de dengue clássico e dos casos ambulatoriais confirmados de dengue com manifestações hemorrágicas.

Do total de 33 casos ambulatoriais clinicamente compatíveis com dengue e apresentando manifestações hemorrágicas, somente 11 (33\%) foram confirmados laboratorialmente como dengue. Estes 11 casos foram classificados como casos de dengue com manifestações hemorrágicas, pois não se enquadram nos critérios adotados pela OMS para o diagnóstico de DH/SCD (o caso 8 apresentou trombocitopenia importante - contagem de plaquetas de $40000 / \mathrm{mm}^{3}$ - mas não apresentou prova do laço positiva ou hemoconcentração na investigação realizada). Os principais tipos de manifestações hemorrágicas observadas foram epistaxe e gengivorragia (55\%). A faixa etária acima dos 20 anos predominou nos casos de dengue com manifestações hemorrágicas (82\%). A totalidade dos casos confirmados ocorreu no mês de dezembro, época do pico da epidemia, sugerindo a perda da sensibilidade do sistema de vigilância nos períodos de menor incidência de casos. Todos os casos de dengue com manifestações hemorrágicas eram do sexo feminino.

A seguir são apresentados dados de três casos hospitalizados que foram investigados como suspeitos de DH/SCD. Chamamos a atenção para os aspectos essenciais encontrados em cada um deles.

O caso 1 mostrou um quadro infeccioso compatível com dengue, peté- quias, sangramento digestivo, prova do laço positiva, trombocitopenia $\left(131000 / \mathrm{mm}^{3}\right)$ e exame sorológico positivo para dengue. Apesar da suspeita inicial de DH/SCD, constituiu-se na realidade um caso de dengue com manifestações hemorrágicas, tomandose como critério a contagem de plaquetas superior a $100000 / \mathrm{mm}^{3}$ (não foi feito hematócrito).

$\mathrm{O}$ caso 2 apresentou um quadro de choque circulatório com história de processo infeccioso compatível com dengue nos 5 dias precedentes. A paciente foi hospitalizada com a impressão diagnóstica de choque séptico, vindo a falecer por colapso circulatório. Somente após o óbito foi levantada pela vigilância epidemiológica a possibilidade de tratar-se de um caso de DH/SCD. Por essa razão, não foi realizado o exame sorológico específico para a investigação etiológica. $\mathrm{O}$ exame necroscópico revelou derrame pleural bilateral e o exame imunohistoquímico realizado em fragmentos de tecidos confirmou a infecção pelo vírus do dengue. Não houve referências a quadro anterior de dengue nem a viagens para regiões endêmicas nos 5 anos precedentes. $\mathrm{O}$ hematócrito encontrado foi de $48 \%$, acima do limite de normalidade para o sexo feminino.

No caso 3 observou-se um quadro de choque circulatório e óbito poucas horas após a hospitalização. Foi formulada a suspeita clínica de choque séptico pelo corpo clínico assistente. A investigação epidemiológica mostrou existir uma história clínica compatível com dengue nos 6 dias precedentes, com queda do estado geral e sudorese fria nas 48 horas anteriores à hospitalização. A investigação revelou também que a paciente havia procurado o centro de saúde de sua cidade já no início da enfermidade, quando se coletou amostra de sangue para investigação laboratorial do dengue. O resultado deste exame, positivo para dengue, só ficou disponível após o falecimento da paciente, devido à demora nos procedimentos laboratoriais e administrativos. Por volta do sexto dia de enfermidade, a paciente apresentou piora e procurou serviço médico-hospitalar, mas foi reencaminhada para sua 
QUADRO 1. Casos ambulatoriais suspeitos de dengue hemorrágico/síndrome de choque do dengue, Ribeirão Preto, São Paulo, $1990-1991^{\mathrm{a}}$

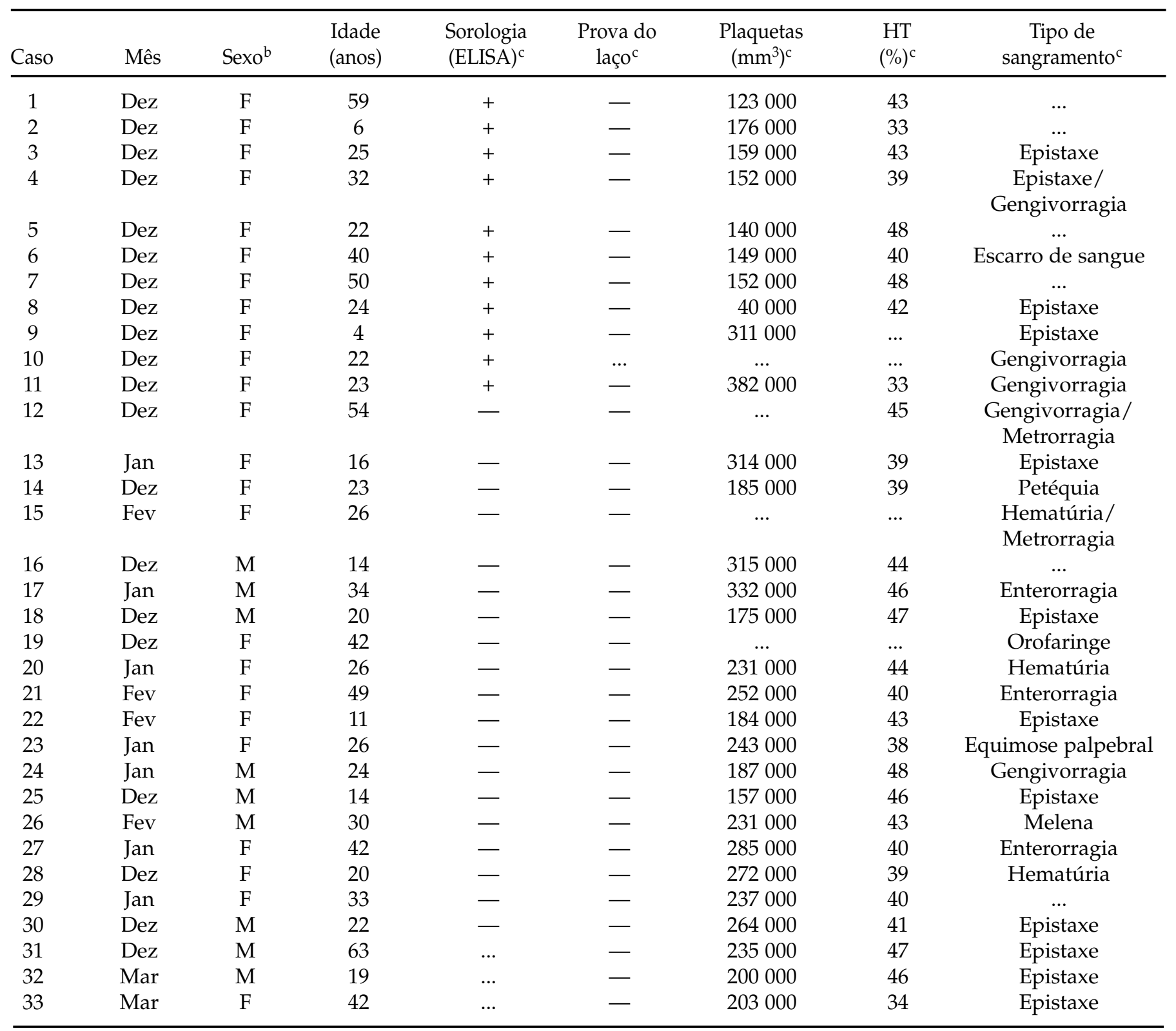

${ }^{a}$ Dados da investigação epidemiológica, Secretaria Municipal da Saúde de Ribeirão Preto.

${ }^{\mathrm{b}} \mathrm{M}=$ masculino; $\mathrm{F}=$ feminino.

c $+=$ positivo; $-=$ negativo; $\ldots$ = não-realizado/sem informação.

residência. Na noite do mesmo dia, a paciente procurou novamente o mesmo serviço e foi hospitalizada por algumas horas. Como não apresentasse melhora, a paciente foi encaminhada para Ribeirão Preto (distante cerca de $20 \mathrm{~km}$ de sua cidade de origem), onde faleceu. A investigação laboratorial realizada mostrou, como dados mais relevantes, evidência de hemoconcentração (hematócrito de $53 \%$ ) e exame sorológico para dengue positivo. O exame necroscópico evidenciou derrame pleural bilateral. A paciente não tinha história anterior de doença compatível com dengue nem deslocamento para fora da região de Ribeirão Preto nos últimos 5 anos.

\section{DISCUSSÃO E CONCLUSÕES}

Como mencionado anteriormente, foram confirmados 12 casos de dengue 
FIGURA 1. Casos ambulatoriais confirmados de dengue clássico e de dengue com manifestações hemorrágicas, Ribeirão Preto, São Paulo, 1990-1991

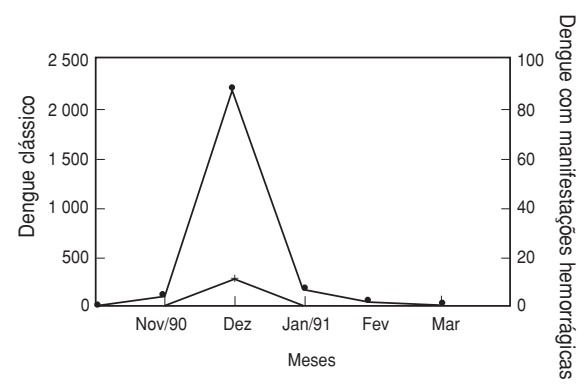

-•- Dengue clássico

-+- Dengue com manifestações hemorrágicas

com manifestações hemorrágicas no Município de Ribeirão Preto (11 provenientes do sistema de vigilância ambulatorial e um caso identificado pelo sistema de busca ativa hospitalar) durante a epidemia de 1990-1991. Isto representou apenas $0,5 \%$ do total de casos de dengue confirmados laboratorialmente no transcorrer do processo epidêmico (12 em 2521 casos). Parte dos resultados negativos encontrados entre os 36 pacientes com suspeita de dengue hemorrágico pode ser conseqüência da época de coleta dos exames laboratoriais, geralmente feita antes do quinto dia da enfermidade, quando são mais freqüentes os resultados falso-negativos. Nos períodos epidêmicos - devido à grande demanda de casos, à sobrecarga do laboratório e ao desinteresse do paciente após a fase aguda da enfermidade - torna-se operacionalmente inviável agendar nova coleta de exame laboratorial para momento posterior ao da primeira consulta, esta geralmente antes do quinto dia de doença.

Da mesma forma, enquanto o coeficiente de incidência do dengue em geral ficou em torno de 557 casos/ 100000 habitantes (2 521 casos confirmados em 452746 habitantes), o coeficiente de incidência do dengue com manifestações hemorrágicas situou-se no patamar de 3 casos/100 000 habitantes (12 casos em 452746 habitantes). Por esses dados, podemos concluir que a ocorrência de manifestações hemorrágicas foi um fenômeno relativamente raro entre os casos identificados através das notificações e busca ativa.

É possível que esses valores não representem a real incidência dessa forma clínica da enfermidade em relação ao total de casos de dengue que procuraram os serviços de saúde, e que não retratem aquilo que ocorreu em nível populacional. Isto porque não havia um sistema de registro e investigação de casos de dengue com manifestações hemorrágicas organizado de forma generalizada nas unidades de saúde municipais (estas unidades apenas encaminhavam o paciente suspeito para o serviço de referência, não realizando investigação clínica e epidemiológica do caso). Tão somente foram notificados e investigados os casos que compareceram ao serviço de referência montado especialmente para dar suporte aos portadores de manifestações hemorrágicas, ou aqueles identificados, através da busca ativa, em hospital universitário. Não é possível, por conseqüência, fazer generalizações a partir dos dados apresentados. Este fato, entretanto, não diminui a relevância epidemiológica de seu registro e divulgação frente à crescente importância do dengue como problema de saúde pública, no Brasil em particular, e nas Américas, de uma maneira geral. Os serviços de saúde pública continuam com uma atitude passiva diante da ocorrência do dengue, em parte como fruto da falta de experiência anterior com a enfermidade. O exemplo de Ribeirão Preto é ilustrativo sobre este aspecto.

Apesar de tratar-se de um município com alto padrão em serviços de saúde, a organização da vigilância e da assistência aos portadores de manifestações hemorrágicas somente foi efetivada com a epidemia já em curso e de forma parcial. É necessário reverter essa perigosa ineficiência e generalizar a prática de vigilância ativa e seletiva sobre os casos de dengue com manifestações hemorrágicas, de modo a possibilitar o diagnóstico diferencial e a detecção precoce de casos de $\mathrm{DH} / \mathrm{SCD}$.
Quanto à predominância do sexo feminino entre os casos confirmados de dengue com manifestações hemorrágicas, podemos levantar a possibilidade de que este achado represente um viés ocasionado pelo horário de funcionamento do ambulatório de referência onde foram investigados os casos (horário diurno, de segunda a sexta-feira). Este horário poderia dificultar o comparecimento das pessoas inseridas no mercado de trabalho, predominantemente do sexo masculino (de acordo com dados da FIBGE (13), cerca de dois terços da população economicamente ativa no Brasil pertencem ao sexo masculino). Outra possibilidade seria uma relação entre sexo e fenômenos hemorrágicos, embora o tipo de estudo realizado não permita nenhuma conclusão a esse respeito.

A análise dos dados referentes aos óbitos investigados mostra que, durante a epidemia de dengue, dois pacientes com quadro clínico característico da enfermidade apresentaram choque circulatório, sem que fosse identificada qualquer ocorrência ou outra doença de base que justificasse a evolução observada. Ambos eram adultos do sexo feminino, fator de risco individual para o DH/SCD (11). Houve confirmação laboratorial de dengue nas duas situações - uma vez através de sorologia e outra por meio de exame imunohistoquímico de fragmento de tecidos. Nos dois casos constatou-se hemoconcentração e também derrame em serosas ao exame necroscópico, um sinal de aumento da permeabilidade vascular no processo evolutivo da enfermidade. Todos estes achados são compatíveis com a definição de caso de DH/SCD preconizada pela OMS (2).

Por outro lado, a exposição anterior daqueles dois pacientes ao dengue possibilitando uma infecção seqüencial - pode ser descartada levando-se em consideração os seguintes aspectos epidemiológicos:

a) a primeira epidemia de dengue registrada no Brasil data de 19811982, tendo ficado circunscrita a uma região geográfica isolada do 
resto do país (Boa Vista, Roraima), localizada na Amazônia brasileira, com circulação de sorotipos DEN-1 e DEN-4 (14). A investigação epidemiológica realizada mostrou que os dois pacientes que evoluíram para óbito nunca estiveram na Região Amazônica.

b) Em 1986-1987 ocorreu nova epidemia de dengue no país, no Rio de Janeiro, centro de intenso fluxo turístico e econômico $(15,16)$. A partir daí, entre 1986 e 1990, diversos outros estados apresentaram processos epidêmicos (como Alagoas, Ceará, Pernambuco, Bahia, Minas Gerais, Mato Grosso do Sul). No Estado de São Paulo também houve um surto de pequenas proporções em 1987 (nas cidades de Guararapes e Araçatuba, situadas numa região cerca de $300 \mathrm{~km}$ distante da região de Ribeirão Preto) (17). Dada a importante circulação do dengue no Brasil neste período, quando passou a apresentar um comportamento endemo-epidêmico na maioria das regiões e áreas geográficas onde foi introduzido, a possibilidade dos dois casos em discussão terem sido expostos ao vírus do dengue anteriormente deveria ser levada em conta. Porém, em todos os processos epidêmicos referidos, ocorridos entre 1986 e 1990 no território brasileiro, houve circulação tão somente do sorotipo DEN-1, o mesmo sorotipo isolado na epidemia de Ribeirão Preto e demais municípios do Estado de São Paulo em 1990-1991. ${ }^{4}$ Este fato por si só afastaria a possibilidade de infecção seqüencial nos casos de DH/SCD

\footnotetext{
4 O sorotipo DEN-2, identificado de forma autóctone pela primeira vez no Brasil em abril de 1990, no Rio de Janeiro (18), provocou uma epidemia cujo pico de maior intensidade se deu a partir de novembro/dezembro de 1990 (19), portanto em época simultânea à epidemia de Ribeirão Preto. Em março de 1991, no estado de Tocantins, ocorreu uma segunda epidemia de DEN-2 no Brasil (20).
}

estudados. Acrescente-se que a investigação epidemiológica realizada mostrou que nenhum deles saiu da região em torno de Ribeirão Preto nos 5 anos anteriores a 1990, e nunca estiveram fora do país, ou seja, não freqüentaram áreas com circulação de dengue.

Em síntese, observamos que durante uma epidemia de dengue em região antes indene à enfermidade, e sem que houvesse antecedentes pessoais de exposição ao dengue em outra área geográfica, dois pacientes apresentaram um quadro compatível com a definição de caso de DH/SCD da OMS. O conjunto de evidências sugere que os dois óbitos investigados eram casos de DH/SCD primário, mesmo não se dispondo de outros dados laboratoriais que melhor caracterizassem uma primo-infecção.

A ocorrência do DH/SCD nas Américas é um fenômeno relativamente recente e parece tratar-se de um problema em evolução. A primeira epidemia dessa forma clínica da enfermidade no continente americano verificou-se em Cuba no ano de 1981 (21). Desde essa época até o ano de 1990 e 1991, quando ocorreu a epidemia de Ribeirão Preto, observou-se o progressivo aumento das notificações do $\mathrm{DH} / \mathrm{SCD}$, com registros tanto de casos isolados em diversos países como também de epidemias, a exemplo da Venezuela (1989-1990) e do Brasil (no Rio de Janeiro, em 1990-1991) $(19,22-25)$. É possível que os níveis elevados de infestação vetorial e a circulação de vários sorotipos virais estejam gestando as condições necessárias para o agravamento do quadro epidemiológico do dengue na região, a exemplo do que ocorreu no sudeste asiático a partir dos anos 50 (26). Por outro lado, pode estar ocorrendo simultaneamente um maior alerta para a busca e notificação dos casos, como decorrência da maior divulgação do problema e disseminação do conheci- mento sobre o dengue, pelo menos entre os profissionais de saúde pública.

No nosso estudo, mostramos que, de fato, a organização de um sistema de vigilância epidemiológica ativo (ainda que parcial), conseguiu identificar casos, tanto de dengue com manifestações hemorrágicas como de DH/SCD, que de outra forma passariam despercebidos. Ao mesmo tempo, evidenciamos a dificuldade da rede assistencial em estabelecer a suspeita diagnóstica de casos de DH/SCD. Lembramos, como um alerta aos serviços de epidemiologia, que nenhum dos dois óbitos por nós estudados foram considerados suspeitos de DH/SCD pelo corpo clínico assistente. Isto aconteceu apesar do quadro clínico bastante sugestivo no decorrer de uma epidemia de dengue. Neste aspecto, o caso 3 serve como uma ilustração eloqüente de como a falta do diagnóstico precoce pode ter concorrido para uma evolução desfavorável: apresentando um evidente quadro de dengue, com riqueza de sinais e sintomas característicos, acrescido de sinais premonitórios de choque na evolução da enfermidade, a paciente procurou atenção médica diversas vezes sem que os serviços hospitalares suspeitassem do diagnóstico. Isto foi agravado por seu encaminhamento a hospital de outro município, retardando ainda mais as medidas terapêuticas necessárias.

É importante chamar a atenção de clínicos e epidemiologistas para ocorrências como estas, que têm no diagnóstico precoce e na intervenção terapêutica imediata os fatores fundamentais para a redução da letalidade. Ao mesmo tempo, dada a expansão do dengue para áreas antes indenes e sua endemização crescente no continente americano, torna-se necessário organizar sistemas permanentes de vigilância e busca ativa de casos de modo a possibilitar a identificação oportuna dos suspeitos de DH/SCD, tanto na forma de casos esporádicos como na forma epidêmica. 


\section{REFERÊNCIAS}

1. Henchal EA, Putnak JR. The dengue viruses. Clin Microbiol Rev 1990;3(4):376-396.

2. Organização Mundial da Saúde. Dengue hemorrágico: diagnóstico, tratamento e controle. Genebra: OMS; 1987.

3. Halstead SB. Pathogenesis of dengue: challenges to molecular biology. Science 1988;239 (4839):476-481.

4. Halstead SB. Antibody, macrophages, dengue virus infection, shock, and hemorrhage: a pathogenic cascade. Rev Infect Dis 1989; 11(suppl 4):S830-839.

5. Kliks SC, Nimmanitya S, Nisalak A, Burke DS. Evidence that maternal dengue antibodies are important in the development of dengue hemorrhagic fever in infants. Am J Trop Med Hyg 1988;38(2):411-419.

6. World Health Organization. Viral haemorrhagic fevers: report of a WHO Expert Committee. Geneva: WHO; 1985. (Technical Report Series 721)

7. Barnes WJS, Rosen L. Fatal hemorrhagic disease and shock associated with primary dengue infection on a Pacific Island. Am J Trop Med Hyg 1974;23(3):495-506.

8. Kuberski T, Rosen L, Reed D, Mataika J. Clinical and laboratory observations on patients with primary and secondary dengue type 1 infections with hemorrhagic manifestations in Fiji. Am J Trop Med Hyg 1977;26(4):775-783.

9. Reed D, Maguirre T, Mataika J. Type 1 dengue with hemorrhagic disease in Fiji: epidemiologic findings. Am J Trop Med Hyg 1977;26(4): 784-791.

10. Kouri GP, Guzman MG, Bravo JR, Triana C. Dengue hemorrhagic fever/dengue shock syndrome: lessons from the Cuban epidemic, 1981. Bull World Health Organ 1989;67(4): 375-380.
11. Kouri GP, Guzman MG, Bravo JR. Why dengue hemorrhagic fever in Cuba? $2-A n$ integral analysis. Trans $\mathrm{R}$ Soc Trop Med Hyg 1977;26(4):784-791.

12. Pontes RJS. Estudo da epidemia de dengue no município de Ribeirão Preto - SP, 1990-1991 [tese de doutorado]. Ribeirão Preto: Faculdade de Medicina de Ribeirão Preto da Universidade de São Paulo; 1992.

13. Fundação Instituto Brasileiro de Geografia e Estatística. Anuário estatístico do Brasil, 1990. Rio de Janeiro: FIBGE; 1990.

14. Osanai $\mathrm{CH}$. A epidemia de dengue em Boa Vista, Território Federal de Roraima, 19811982 [dissertação de mestrado]. Rio de Janeiro: Escola Nacional de Saúde Pública; 1984.

15. Schatzmayr HG, Nogueira RMR, Travassos da Rosa APA. An outbreak of dengue virus at Rio de Janeiro. Mem Inst Oswaldo Cruz 1986;81(2): 245-246.

16. Nogueira RMR, Schatzmayr HG, Miagostovich MP, Farias MFDB, Farias Filho JC. Virological study of a dengue type 1 epidemic at Rio de Janeiro. Mem Inst Oswaldo Cruz 1988; 83(2):219-225.

17. Pontes RJS, Ruffino-Netto A. Dengue em localidade urbana da região sudeste do Brasil: aspectos epidemiológicos. Rev Saude Publ 1994;28(3);218-227.

18. Nogueira RMR, Miagostovich MP, Lampe E, Schatzmayr HG. Isolation of dengue virus type 2 in Rio de Janeiro. Mem Inst Oswaldo Cruz 1990;85(2):253.

19. Theme Filha M, Baran M, Gama SGN, Silva D. O dengue no município do Rio de Janeiro - 1990-1991 - o dengue hemorrágico. Em: Resumos do II Congresso Brasileiro de Epidemiologia. Belo Horizonte: ABRASCO; 1992:62.
20. Vasconcellos PFC, Travassos da Rosa ES, Travassos da Rosa JFS, Freitas RB, Dégallier N, Rodrigues SG, Travassos da Rosa APA Epidemia de febre clássica de dengue causada pelo sorotipo 2 em Araguaiana, Tocantins, Brasil. Rev Inst Med Trop Sao Paulo 1993;35(2): 141-148.

21. Kourí G, Guzmán MG, Bravo J. Dengue hemorrágico en Cuba: crónica de una epidemia. Bol Oficina Sanit Panam 1986;100(3): 322-329.

22. Lopez-Correa RH, Cline BL, Ramirez-Ronda C, Bermudez R, Sather GE, Kuno G. Dengue fever with hemorrhagic manifestations: a report of three cases from Puerto Rico. Am J Trop Med Hyg 1978;27(6):1216-1224.

23. Organización Panamericana de la Salud. El dengue y la fiebre hemorrágica de dengue en las Américas: una visión general del problema. Bol Epidemiol 1992;13(1):9-10.

24. Pinheiro FP, Communicable Diseases Program, Pan American Health Organization. Dengue in the Americas, 1980-1987. Epidemiol Bull 1989;10(1):1-8.

25. Pan American Health Organization. Dengue hemorrhagic fever in Venezuela. Epidemiol Bull 1990;11(2):7-9.

26. Halstead SP. Global epidemiology of dengue hemorrhagic fever. Southeast Asian J Trop Med Public Health 1990;21(4):636-641.

Manuscrito recebido em 9 de outubro de 1995. Aceito para publicação em versão revisada em 15 de maio de 1996.

ABSTRACT A clinical-epidemiological case study was carried out to evaluate a surveillance and active search system for suspect cases of dengue hemorrhagic fever/dengue shock syndrome (DHF/DSS) during a dengue epidemic in the Ribeirão Preto municipality,

\section{Surveillance and active search system for suspect cases of hemorrhagic dengue in Ribeirão Preto, São Paulo} State of São Paulo, Brazil, between November 1990 and March 1991. This was the first dengue epidemic ever recorded in this region. Although dengue is a growing public health problem in Brazil, and DHF/DSS has been reported to be evolving in the Americas, health officials have shown a passive attitude towards the disease, partly due to lack of experience. Reporting dengue occurrences, both of isolated cases and of epidemics, is essential to reduce mortality.

The data analyzed were collected through the official epidemiological surveillance system during the 1990-1991 epidemic. Out of the 2521 confirmed cases of dengue, 34 cases of febrile acute illness with hemorrhagic manifestations were identified as possible cases of DHF/DSS, as well as two deaths associated to dengue. From these 36 cases, 12 cases were confirmed by laboratory exams as dengue with hemorrhagic manifestations. Examination of clinical and laboratory data, and results of the autopsies suggested that the two deaths were cases of primary DHF/DSS. The difficulty of the health system in establishing the clinical suspicion of DHF/DSS was confirmed in the study. This resulted in delayed and inadequate clinical procedure. The present study also confirmed the need for a permanent active epidemiological surveillance system for the early identification of DHF/DSS suspect cases thus enabling quick and adequate control actions. 